\title{
Descompresión medular e instrumentación en un caso de variante sólida de quiste óseo aneurismático en raquis lumbar
}

\author{
A.A. Díaz-Martín; N. Guerrero-Moyano; E. Guerado-Parra y J.A. Quesada-Rubio*
}

Hospital Costa del Sol. Marbella. *Hospital Niño Jesús. Madrid.

\section{Resumen}

Se discute el caso de un paciente varón de ocho años de edad que refería dolor y limitación funcional en extremidad inferior izquierda. Tras estudios complementarios mediante radiografías, tomografía axial computarizada, resonancia magnética y gammagrafía se evidenció la presencia de una lesión osteolítica en cuerpo y arco posterior de la tercera vértebra lumbar. La tumoración fue resecada a través de doble abordaje (anterior retroperitoneal y posterior) e instrumentación pedicular posterior con aporte estructural de hueso tricortical liofilizado en un solo tiempo. El diagnóstico histológico reveló la presencia de variante sólida de un quiste óseo aneurismático. Cinco años tras la cirugía el paciente no presentó dolor lumbar ni recurrencia de la enfermedad. La resección completa del tumor junto con instrumentación pedicular previno recidivas y desarrollo de inestabilidad.

PALABRAS CLAVE: Columna lumbar. Quiste óseo aneurismático. Artrodesis lumbar.

Spine cord descompresion and instrumentation in a case of solid aneurysmal bone cyst in the lumbar spine

\section{Summary}

We report a 8-year-old boy with two month history of pain and stiffness in his left lower extremity. On $x$ ray, MRI and scintigraphy examinations a lytic lesion affecting the body and posterior arc of his third lumbar vertebral become evident. The tumour was excised through a double, anterior and posterior approach; spinal fusion with posterior transpedicular instrumentation was also performed in the same procedure; in order to achieve solid fusion tricortical liofilized graft was also inserted. Histological studies evidenced finding compatible with aneurismal bone cyst solid variant.

Recibido: 29-09-09. Aceptado: 16-11-09
Five years after surgery the patient is pain free and no recurrence is evident under diagnostic images tools. For the treatment of aneurismal bone cyst of the spine we recommend complete excision of the tumour in a combined anterior and posterior one staged procedure, together with hardware instrumentation and bone graft, no recurrence or instability is seen after five years followup.

KEY WORDS: Lumbar spine. Aneurysmal bone cyst. Spinal stabilization

\section{Introducción}

El quiste óseo aneurismático constituye el $1 \%$ de los tumores óseos, se localiza en raquis lumbar en el $25 \%$ de los $\operatorname{casos}^{5}$. Está considerada como una lesión tumoral ósea benigna pero con agresividad local osteolítica, expansiva y de etiología desconocida. Esta lesión suele aparecer en la infancia y adolescencia presentando predilección por elementos posteriores vertebrales. El tumor puede invadir a estructuras óseas adyacentes y en un $45 \%$ de los casos invadir cuerpo vertebral ${ }^{13}$. Existe un porcentaje elevado de los mismos de transmisión familiar ${ }^{8}$.

Parece haber un consenso sobre el tratamiento de estas lesiones cuando afecta a la columna vertebral ${ }^{7}$, diferente de los protocolos estandarizados en el tratamiento de estas lesiones líticas localizadas en otras regiones ${ }^{2}$. Debido a su potencial capacidad de invasión y destrucción de más de un nivel vertebral con extensión intraespinal, provocando compresión extradural con aparición de signos y síntomas de compromiso neurológico, la resección completa de la lesión es importante para lograr el éxito del tratamiento quirúrgico. Cuando están afectados múltiples segmentos, el procedimiento terapéutico estándar de curetaje e injerto óseo puede no ser adecuado por que podría provocar inestabilidad de raquis con el riesgo de desarrollar compromiso neurológico ${ }^{16}$.

Abreviaturas. RM: Resonancia magnética nuclear. TC: tompografía axial computarizada. Tc-99: tecnesio 99. 


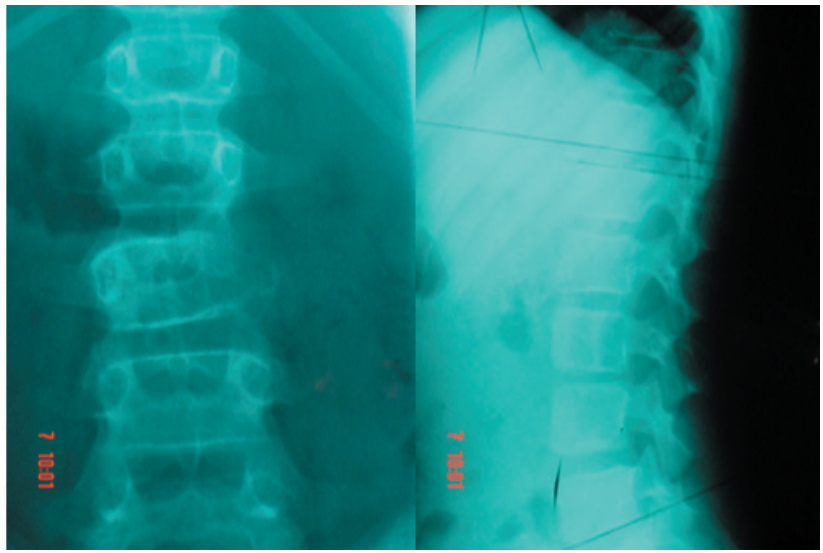

Figura 1. Imagen radiológica donde se aprecia una lesión osteolítica expansiva ocupando la porción posterior del cuerpo vertebral y pedículo izquierdo, provocando escoliosis reactiva y rectificación de lordosis fisiológica lumbar.

En este trabajo se presenta el resultado de un quiste óseo aneurismático localizado en raquis lumbar, tras practicar resección completa y estabilización quirúrgica. Por su infrecuencia y posibilidad de recidiva debido a su agresividad local se discute el tratamiento tras cinco años de seguimiento.

\section{Caso clínico}

Niño de ocho años de edad que presentaba dolor muy intenso en región dorso-lumbar de dos meses de evolución sin antecedente traumático previo, así como pérdida de fuerza a la extensión de la rodilla izquierda. La exploración clínica evidenció la presencia de dolor a la palpación de apófisis espinosas desde segunda a cuarta vértebra lumbar con limitación de flexo-extensión de raquis lumbar, contractura paravertebral musculatura lumbar y déficit de extensión contra resistencia rodilla izquierda.

El estudio radiológico reveló la presencia de una lesión lítica afectando la porción posterior del cuerpo vertebral de la tercera vértebra lumbar, con lisis de su pedículo izquierdo y colapso parcial vertebral sin deformidad residual (Fig.1).

Tras realizar tomografía axial computarizada se confirmó que la lesión estaba limitada al cuerpo, pedículo y lámina izquierda de la tercera vértebra lumbar (Fig.2). En imágenes de resonancia magnética se apreció una tumoración expansiva isointensa en secuencia T1 en tercera vértebra lumbar con masa de partes blandas obliterando raíces en el lado izquierdo y el receso foraminal ipsilateral e invasión del canal raquídeo. Las imágenes en secuencia T2 muestran señales hipointensas (Fig.3).

La gammagrafía con Tc-99 y citrato de Galio 67 reveló discreta hipercaptación del trazador en L3 y L4. Tras seis

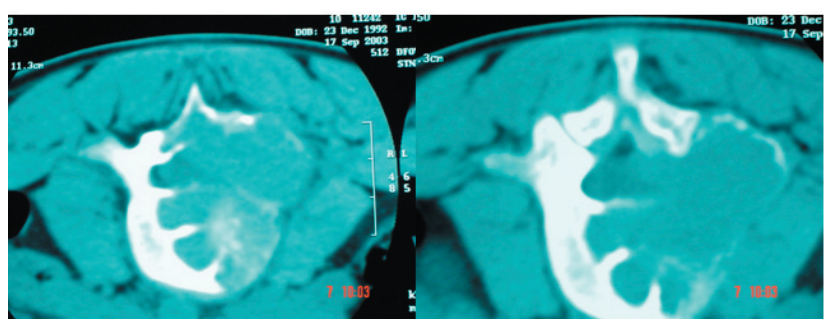

Figura 2. Imagen tomografía axial computarizada que evidencia la presencia de una lesión osteolítica en el cuerpo vertebral e invade el canal neural, destruye cortical vertebral y desplaza el saco neural, afectando tejidos blandos.

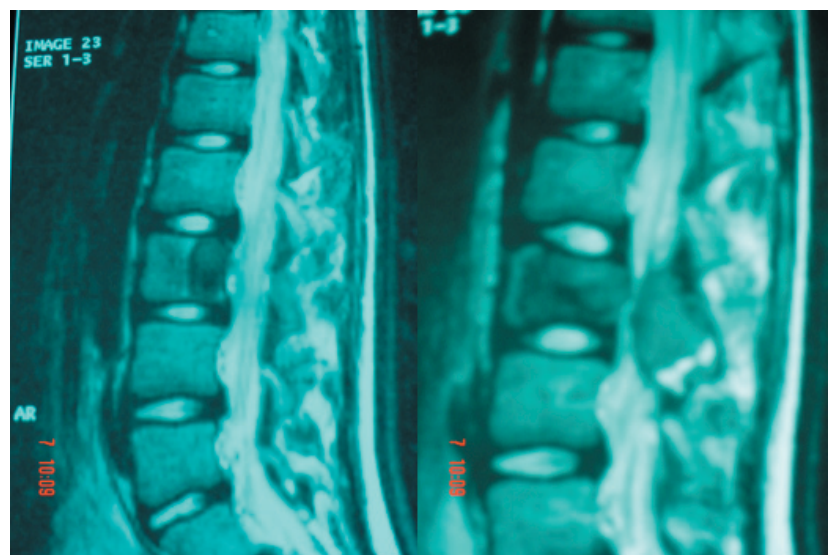

Figura 3. Imagen de resonancia magnética en corte sagital (A): potenciada en $T 2$ revela la presencia de una masa homogénea e isointensa que afecta a cuerpo posterior vertebral sin afectación espacio discal. (B): masa tumoral homogénea que afecta a elementos posteriores de vértebra lumbar provocando grave estenosis del canal, receso lateral izquierdo y rectificación de la lordosis lumbar.

días de administración del contrate se realizó nuevo estudio, confirmándose ligera captación del trazador en las zonas sin ninguna alteración a otro nivel.

Se practicó intervención quirúrgica, posicionando al paciente en decúbito prono, mediante incisión en línea media a nivel lumbar, realizando escisión de elementos posteriores de la tercera vértebra lumbar e instrumentación con tornillos pediculares en segunda y cuarta vértebra lumbar; seguidamente se realizó lumbotomía derecha, corpectomía de la vértebra afecta y aporte biológico sustitutivo del cuerpo vertebral con injerto óseo liofilizado tricortical. (Fig.4).

El estudio histológico reveló la presencia de un quiste óseo aneurismático de tipo sólido con presencia de células con morfología fibrohistiocitarias, junto a células inflamatorias en un estroma ricamente vascularizado y pseudotrabéculas de aspecto osteoide (Fig.5).

Tras cinco años de seguimiento el paciente se encuentra asintomático sin signos de recidiva ni de inestabilidad clí- 


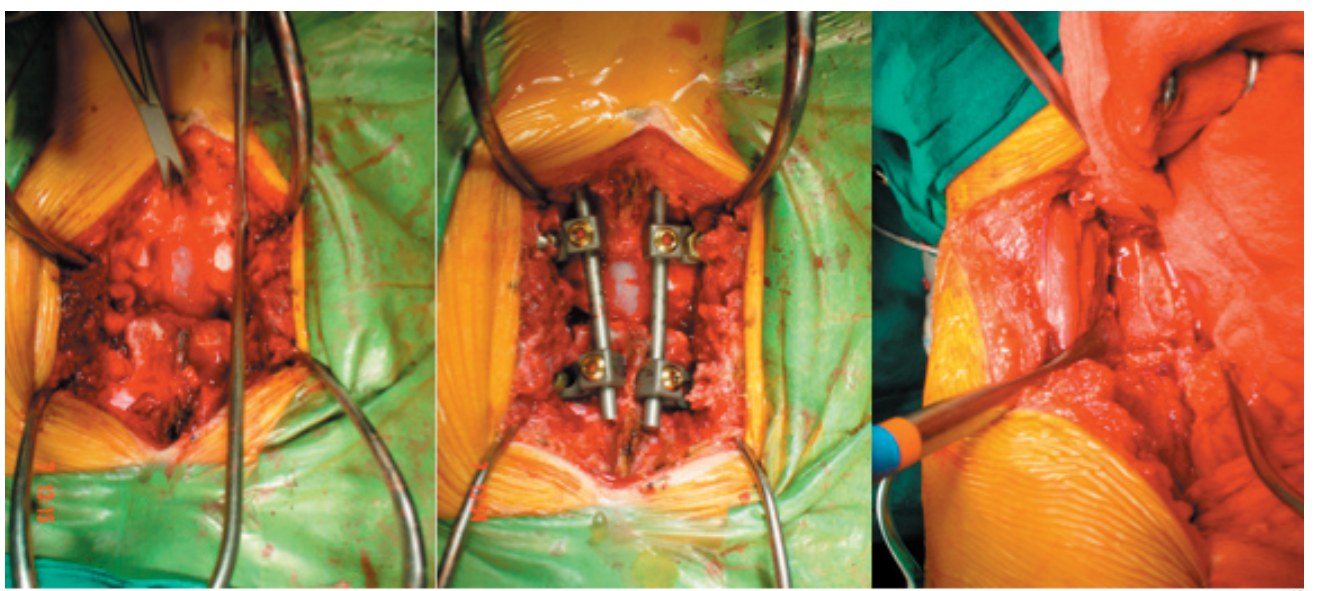

Figura 4. Imágenes intraoperatorias: A): resección del arco posterior de la tercera vértebra lumbar $y$ descompresión del saco neural. B): aplicación de fijador vertebral. C): tras realizar corpectomia se procede a la aposición de injerto estructural tricortical liofilizado intersomático.

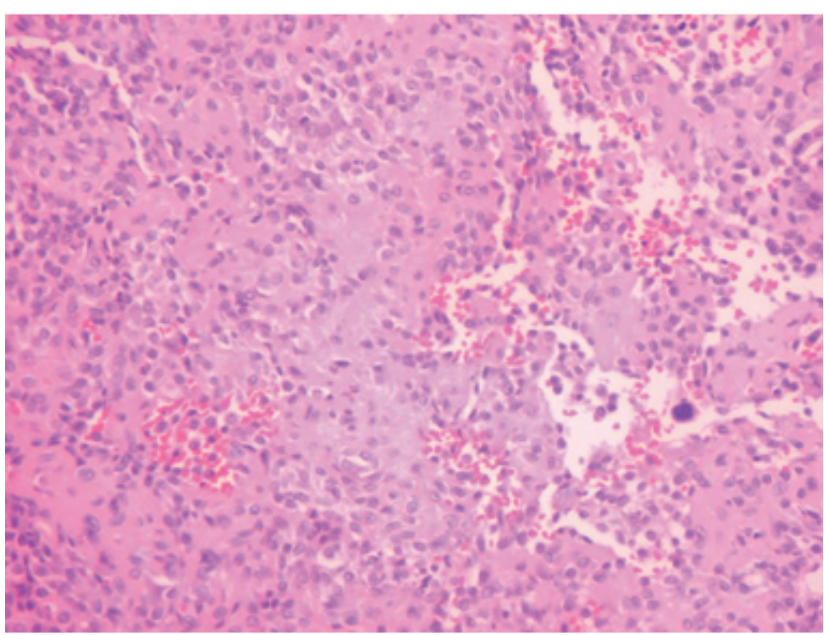

Figura 5. Hallazgos anatomopatológicos de la tumoración: presencia de células con morfología fibrohistiocitarias y núcleos aumentados de tamaño con pequeñas cavidades, junto a células inflamatorias en un estroma vascularizado.

nica ni radiológica (Fig.6).

\section{Discusión}

El quiste óseo aneurismático se define como una lesión tumoral benigna, vascularizada, de agresividad local y etiología desconocida, compuestas de espacios cavernosos ocupados por sangre con un estroma muy celular compuesto de fibroblastos y células gigantes semejantes a osteoclastos delimitados por septos fibrosos ${ }^{15}$. Debido a la velocidad de crecimiento presenta riesgo elevado de provocar fractura patológica ${ }^{4}$.

La localización de la lesión en el esqueleto axial ocurre en un $25 \%$ de los casos, afectándose el arco vertebral posterior con más frecuencia que los cuerpos vertebrales, y especial incidencia en niños y adolescentes, sobre todo en el sexo femenino ${ }^{13}$. Esta lesión lítica puede, además, invadir

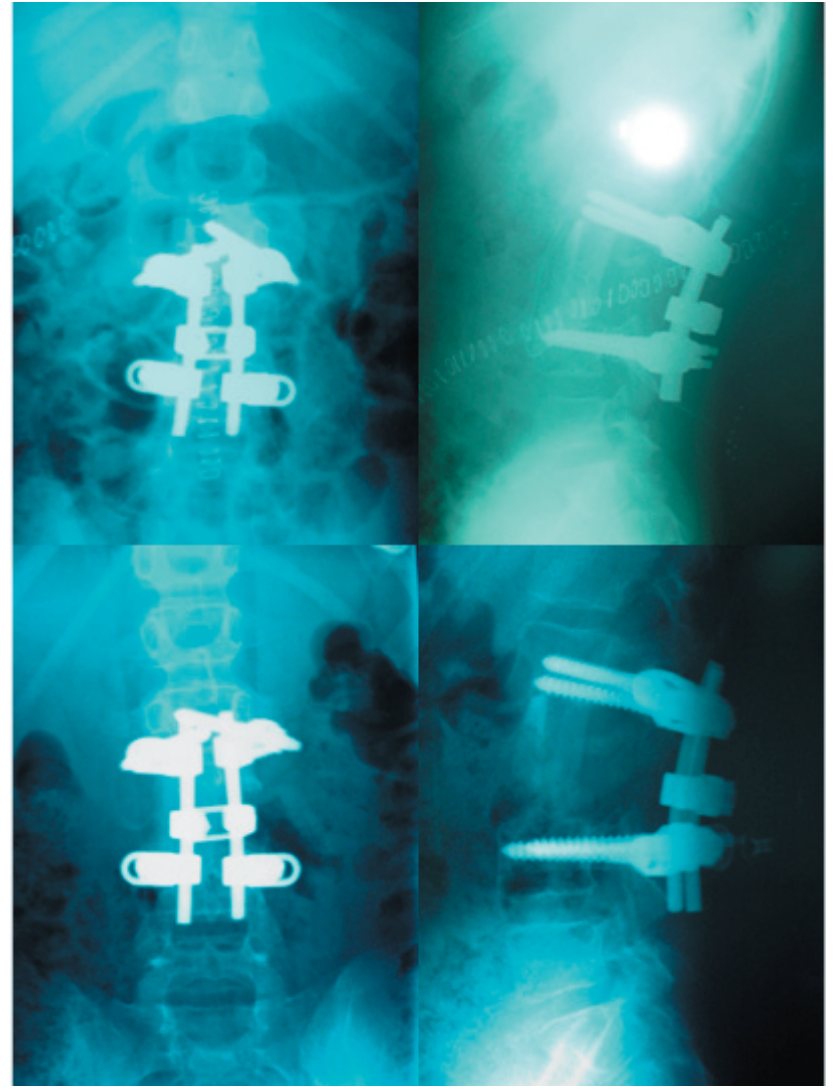

Figura 6. Aspecto radiologico: A): imagen postoperatoria: se aprecia instrumentación transpedicular, aporte de injerto estructural, conservación de espacio vertebral y conservación de lordosis lumbar. B): cinco años postcirugía se muestra artrodesis y fusión intersomática anterior sin evidencia de recidiva local ni movilización del instrumental.

y destruir estructuras óseas contiguas y producir invasión intraespinal y sintomatología neurológica ${ }^{16}$, La variante sólida es infrecuente presentando, precisamente, predilección por el esqueleto axial, correspondiendo sólo al $6 \%$ de 
los quistes óseos aneurismáticos ${ }^{1}$. Cuando esta lesión se presenta en raquis puede ser malinterpretada como lesión maligna, sobre todo cuando se asocia a colapso del cuerpo vertebral o se afectan las facetas articulares.

Las manifestaciones clínicas son diversas, como dolor, contractura muscular, escoliosis postural y signos neurológicos. La radiología simple en la variante sólida muestra lesión excéntrica lítica que insufla la cortical, pudiendo mostrar la presencia de trabéculas en el interior de la tumoración, resultando difícil diferenciar si la lesión es o no benigna ${ }^{1,6}$ así como escoliosis postural reactiva debida a la contractura de la musculatura paravertebral.

La TC no aporta hallazgos patognomónicos y destaca en la forma clásica la presencia de una lesión osteolítica con fractura de la cortical y afectación de partes blandas bien definida, a diferencia de la variante sólida en la que suele aparecer lesión expansiva osteolítica, rotura de la cortical, afectación de tejidos blandos, desplazamiento de estructuras neurales, calcificación en la zona central del tumor y zona de transición entre el hueso sano y el tumor ${ }^{4,6}$. La $\mathrm{RM}$ en la forma clásica muestra septos en el interior de la tumoración con contenido hemático y márgenes delimitados. La variante sólida no muestra estos septos, destacando masa homogénea, bien circunscrita e isointensa en T1 y heterogénea en T2, sin apreciarse niveles de fluido en su interior, resultando imposible realizar diagnostico diferencial con lesiones tales como sarcoma Ewing, osteoblastoma o tumor de células gigantes ${ }^{17}$. La ausencia de signos patognomónicos radiológicos de esta variante obliga a realizar su diagnostico mediante estudio anatomopatológico.

La primera descripción histológica de la variante sólida corresponde a Sanerkin ${ }^{14}$, comunicando la presencia de múltiples fibroblastos, células inflamatorias multinucleadas, con estroma escaso y ricamente vascularizados con ausencias de lagos vasculares lo que permite realizar diagnóstico diferencial de la variante sólida, junto a pseudotrabéculas osteoides con pequeños núcleos de proliferación osteoclástica, sin atipia celular, permitiendo esto diferenciarlo de procesos malignos. La presencia de calcificación en el estroma puede confundirlo con el condroblastoma. En el estudio histológico se encuentran elevado número de células en actividad mitótica que obligan a realizar diagnóstico diferencial con lesiones de naturaleza sarcomatosa. La ausencia de atipia celular, la presencia de células proliferativas derivadas del linaje osteoblástico, estroma con matriz fibrosa en la que se encuentran incluidos pequeños capilares aneurismáticos, a diferencias de los lagos de la variante clásica del quiste óseo aneurismático, permiten realizar su diagnostico histopatológico. Igualmente pueden aparecer focos de osteoide y depósitos de hemosiderina. Los estudio de inmunohistoquímica demuestran positividad para vimentina y negatividad para citoqueratina $^{1,4,14}$.
Existen controversias actuales a la hora de plantear el tratamiento cuando la tumoración se localiza en el esqueleto axial. La práctica de simple curetaje con aporte de injerto en la lesión se asocia con cifras elevadas de recidivas que pueden llegar hasta el $20 \%$ en la variante clasica ${ }^{17}$, mientras que la variante sólida en raquis no suele presentar recidivas tras resección ${ }^{3}$.

El tratamiento radioterápico se encuentra en discusión debido a que se han encontrado casos que han desarrollado osteosarcomas postradiación ${ }^{4,10}$. Por lo tanto es difícil justificar el uso de un agente carcinógeno potencial ante una lesión benigna cuando han sido utilizados otros métodos de tratamiento adyuvante como la embolización arterial en el tratamiento de tumores en región torácica y lumbar con resultados satisfactorios ${ }^{9,12}$.

La embolización selectiva es eficaz en casos de difícil acceso, excepto en aquéllos que presentan fracturas patológicas o síntomas neurológicos, en los que es necesario realizar cirugía ${ }^{2,11}$. A la hora de llevar a cabo el tratamiento quirúrgico se debe tener en consideración los siguientes factores: localización y extensión de la lesión, estabilidad de la columna y posible desarrollo de complicaciones neurológicas.

El objetivo del tratamiento quirúrgico es lograr una completa escisión del tumor, restablecer la función neurológica y estabilizar la columna vertebral. En el caso que presentamos la tumoración afectaba a elementos anteriores y posteriores vertebrales, provocando inestabilidad y déficit neurológico. La resección tumoral completa, con descompresión medular y estabilización mecánica evita la recidiva e inestabilidad tras cinco años de seguimiento.

\section{Bibliografía}

1. Bertoni, F., Bacchini, P., Ruggieri, P., Biagini, R., Ferruzzi, A., Bettelli, G., el al.: Solid variant of aneurismal bone cyst. Cancer 1993; 71: 729-734.

2. Boriani, S., De Iure, F., Campanacci, L., Gasbarrini, A., Bandiera, S., Biagini, R., et al.: Aneurysmal bone cysts of the mobile spine: report on 41 cases. Spine 2001 Jan 1; 26: 2735.

3. Chiriboga Accini, C., Pérez Domínguez, M., Veras del Monte, L., López, C., Castellanos Robles, J., Mateo Montañes, X.: Quiste óseo aneurismático. Rev Ortop Traumatol 1997; 41: 594-599.

4. Claus Peter, A.: Solid aneurismal bone cyst with pathologic bone fracture. Skeletal Radiol 1995; 24: 214-216.

5. Cottalorda, J., Kohler, R., Sales de Gauzy, J., Chotel, F., Mazda, K., Lefort, G., et al.: Epidemiology of aneurismal bone cyst in children: a multicenter study and literature review. Journal of Pediatric Orthopaedics B 2004; 13: 389-394.

6. Dahlin, D.C., Mcleod, R.: Aneurismal bone cyst and other non neoplastic conditions. Skeletal Radiol 1982; 8: 243- 
250.

7. De Kleuver, M., van der Heul, R.O., Veraart, B.E.: Aneurysmal bone cysts of the spine: 31 cases and the importance of the approach. J Pediatr Orthop B. 1998 Oct; 7: 286292.

8. Di Caprio, M.R., Murphy, M.J., Camp, R.L.: Aneurysmal bone cysts of the spine with familial incidence. Spine 2000 Jun 15; 25: 1589-1592.

9. Dubois, J., Chigot, V., Grimard, G., Isler, M., Garel, L.: Sclerotherapy in aneurysmal bone cysts in children: a review of 17 cases. Pediatr Radiol. 2003 Jun; 33: 365-372.

10. Feigenberg, S.J., Marcus, R.B., Zlotecki, R.A., Scarborough, M.T., Berrey, B.H., Enneking, W.F.: Megavoltage radiotherapy for aneurysmal bone cysts. Int $\mathrm{J}$ Radiat Oncol Biol Phys. 2001 Apr 1; 49: 1243-1247.

11. López Martín, L., Álvarez Galovich, Calvo Crespo, E., Bouhajeb, Y.: Variante sólida de quiste óseo aneurismático en raquis. Rev Ortop Traumatol 2004; 48: 370-374.

12. Meyer, S., Reinhard, H., Graf, N., Kramann, B., Schneider, G.: Arterial embolization of a secondary aneurysmatic bone cyst of the thoracic spine prior to surgical excision in a 15 years old girl. Eur J Radiol. 2002 Jul; 43: 79-81.

13. Papagelopoulos, P.J., Currier, B.L., Shaughnessy, W.J., Sim, F.H., Ebsersold, M.J., Bond, R., et al.: Aneurismal bone cysts of the spine. Management and outcome. Spine 1998 Mar
$1 ; 23 ; 621-628$.

14. Sanerkin, N.G., Mott, M.G., Roylance, J.: An unusual intraosseous lesion with fibroblastic, osteoclastic, osteoblastic, aneurysmal and fibromixoid elements. Solid variant of aneurismal bone cyst. Cancer1983; 50: 2278-2286.

15. Sato, K., Sugiura, H., Yamamura, S., Takahashi, M., Nagasaka, T., Fukatsu, T.: Solid variante of an aneurismal bone cyst (giant cell reparative granuloma) of the $3^{\text {rd }}$ lumbar vertebra. Nagoya J Med Sci 1996; 59: 159-165.

16. Turker, R.J., Mardjetko, S., Lubicky, J.: Aneurysmal bone cysts of the spine: excision and stabilization. J Pediatr Orthop. 1998 Mar-Apr; 18: 209-213.

17. Weinstein, J.B., Siegel, M.J., Griffith, R.: Spinal Ewing sarcoma: misleading appearances. Skeletal Radiol 1984; 11: 262-265.

Díaz-Martín, A.A.; Guerrero-Moyano, N.; Guerado-Parra, E.; Quesada-Rubio, J.A.: Descompresión medular e instrumentación en un caso de variante sólida de quiste óseo aneurismático en raquis lumbar. Neurocirugía 2010;21: 240-244.

Correspondencia: Aurelio Andrés Díaz Martín. Servicio de Cirugía Ortopédica y Traumatología. Hospital Costa del Sol. Ctra. Nacional 340, Km. 187. 29670 Marbella. Málaga.

E-mail: aureliodiazm@yahoo.es 